\title{
Tricky Registration for Unruly Data: Image Registration of Low-Signal-to-Noise Cryo-STEM Data
}

Benjamin H. Savitzky ${ }^{1}$, Ismail El Baggari ${ }^{1}$, Colin Clement ${ }^{1}$, Emily Waite ${ }^{2}$, Robert Hovden ${ }^{2}$, John P. Sheckelton $^{3}$, Christopher Pasco ${ }^{3}$, Tyrel M. McQueen ${ }^{3}$, Alemayehu S. Admasu ${ }^{4}$, Jaewook Kim ${ }^{4}$, SangWook Cheong ${ }^{4}$ and Lena F. Kourkoutis ${ }^{2,5}$

1. Department of Physics, Cornell University, Ithaca, NY.

2. School of Applied \& Engineering Physics, Cornell University, Ithaca, NY.

3. Department of Chemistry, The Johns Hopkins University, Baltimore, MD.

4. Rutgers Cent. Emerg. Mater. and Dept. of Phys. and Astr., Rutgers U., Piscataway, NY.

5. Kavli Institute for Nanoscale Science, Cornell University, Ithaca, NY.

Acquiring stacks of images, which can ultimately be aligned and averaged in post-processing, has become an increasingly important tool for high precision, quantitative analysis of STEM data $[1,2,3]$. However, oftentimes stringent data acquisition requirements can result in images that are difficult to register due to low signal-to-noise ratios (SNRs). For instance, while cryogenic STEM imaging enables characterization of previously inaccessible samples, it also introduces additional stage instability. This challenge can be surmounted through fast acquisition image stacks, however, the SNR of the resulting data is generally quite low (Fig. 1a-c). Similarly, low SNRs may be unavoidable when imaging beam sensitive samples or performing other in-situ measurements. In these cases, established approaches to image registration can fail, thus requiring new approaches for successful data registration.

Here, we present an image registration approach that is optimized for difficult, low-SNR cryo-STEM images, which often cannot be registered successfully by other means, achieving high-SNR average images with $0.72 \AA$ information transfer at cryogenic temperatures (Fig. 1d-f) [4]. We introduce an approach which does not rely on a single reference image, but instead uses all possible combinations of image correlations to determine the optimal shifts. This results in an information surplus, enabling enforcement of physical consistency between all measured relative image shifts. Consequently, incorrect correlations, which plague low-SNR data, may be identified and handled (Fig. 2). Moreover, the criterion of physical consistency may be used to substantiate the validity of the registration.

Importantly, the likelihood of incorrect, and in some cases subtly specious, final image reconstructions due to registration error will vary from one dataset to the next. Lattice images constitute a notable class of images requiring special care, as highly translationally symmetric data has a propensity to register incorrect 'unit-cell jumps'. Tools to avoid or account for these and other errors will be discussed, such as judicious Fourier space weighting, or preemptive avoidance of sampling errors (Fig. 3).

To facilitate user accessibility, all associated code is open source, and may be conveniently run through either the iPython/Jupyter notebook or a graphical user interface. Practical matters for optimal user experience will be discussed and briefly demonstrated. [5]

References:

[1] X Sang, \& JM Lebaeu, Ultramicroscopy 138 (2014), p. 28.

[2] B Berkels et al, Ultramicroscopy 138 (2014), p. 46.

[3] C Ophus, J Ciston, \& CT Nelson, Ultramicroscopy. 162 (2016), p. 1.

[4] BH Savitzky et al, arXiv:1710.09281 (2017).

[5] BHS acknowledges support from the NSF GRFP (DGE-1144153). This work was supported by NSF (DMR-1539918, DMR-1429155, DMR-1719875) and DOD AFOSR (FA 9550-16-1-0305). 

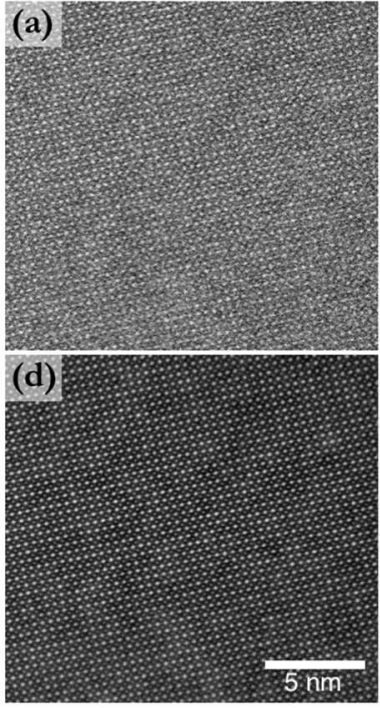
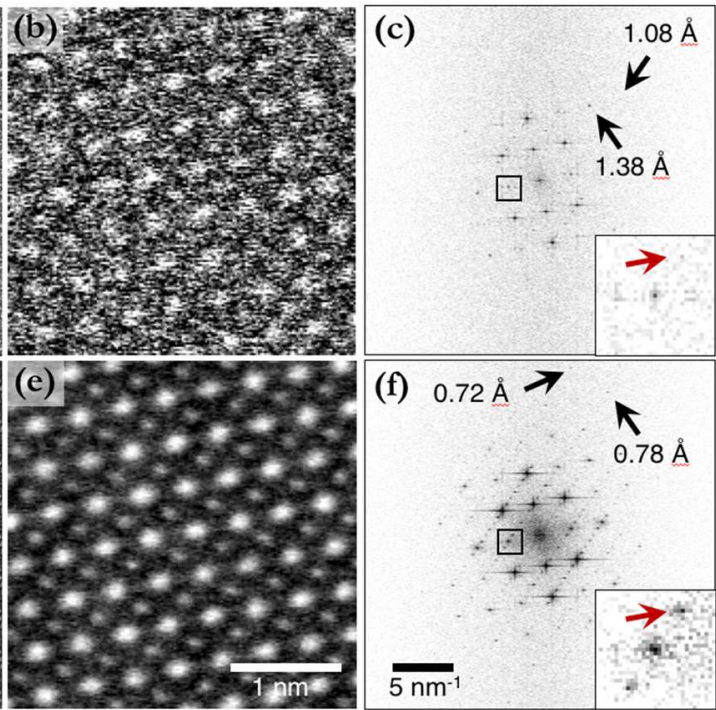

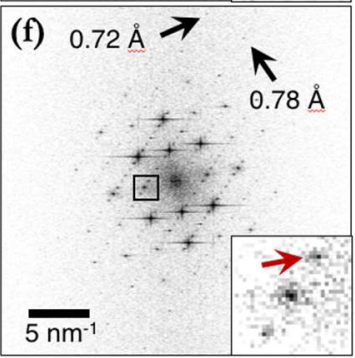

Figure 1. Registration of low SNR cryo-STEM data. (a-c) A single fast acquisition $(0.63 \mathrm{~s})$ frame from a 40 image series of a $\mathrm{Bi}_{1-x} \mathrm{Sr}_{\mathrm{x}-\mathrm{y}} \mathrm{Ca}_{\mathrm{y}} \mathrm{MnO}_{3}$ (BSCMO) crystal imaged under liquid nitrogen cooling. In the full field of view (a) and zoom-in (b) the brighter A-sites of the perovskite lattice are identifiable but noisy, while the dimmer B-sites cannot be clearly distinguished. The FFT (c) shows clear information transfer to $1.38 \AA$, and very weak reflections at $1.08 \AA$. (d-f) Registering and averaging all 40 images by determining the optimal shifts from all image pairs yields a high SNR final image (a), clearly identifiable A- and B-sites (b), and information transfer to $0.72 \AA$ (c). BSCMO hosts a charge density wave state which breaks translational symmetry, manifesting as satellite peaks in the FFT (red arrows), which are undetectable in a single frame but are immediately apparent in the registered data.

Figure 2. Referenceless cryo- (a)

STEM image correlation. (a) All possible image pairs in a stack of fast acquisition cryoSTEM images are cross correlated to determine their relative shifts. (b) The shift matrices $\quad \boldsymbol{R}_{i j}=\boldsymbol{X}_{i j}+\boldsymbol{Y}_{i j}$ visualize the calculated shifts between all image pairs $(i, j)$ (left), from which the optimum (b)

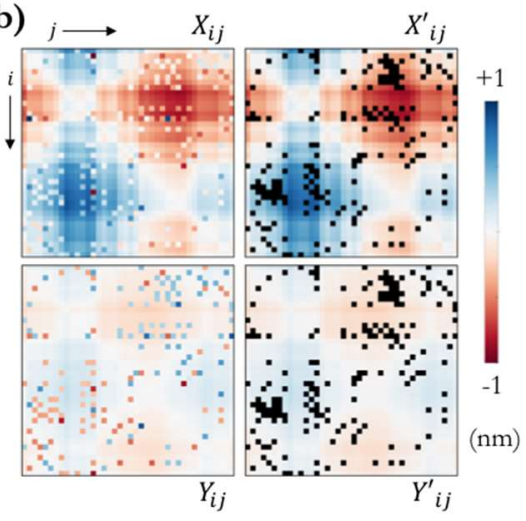

(c)
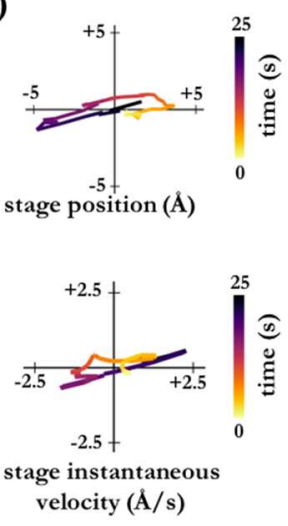

global image shifts may be calculated. The smoothly varying background encodes stage movement during acquisition (c), while the aberrant pixels are incorrect correlations, resulting from the low-SNR of the images. False correlations can thus be identified (b, right) and corrected.

Figure 3. Unit cell jumps and sampling error. (a) The cross correlation between a pair of atomic resolution cryo-STEM images contains many local maxima, corresponding to crystal lattice vector offsets between the images. (b) In this case, the correct maximum is highlighted by the black box, however, the brightest pixel is in the local maximum in the red box, due to sampling effects. Extra care is therefore required to avoid "unit cell hopping" during registration. (c) Here, Gaussian fits to multiple local maxima in the cross correlation reduces erroneous shift matrix elements by $50 \%$.
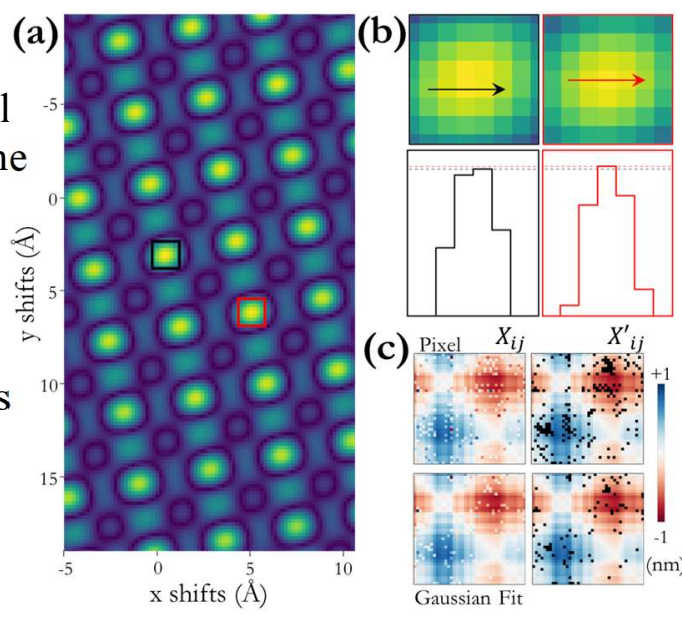

(c)

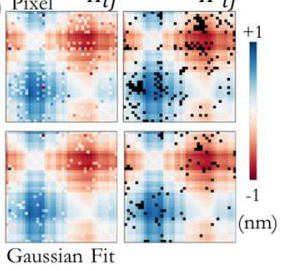

